\title{
Resistência mediada por aleloquímicos de genótipos de tomateiro à mosca-branca e ao ácaro-rajado
}

\author{
Vanisse de Fátima Silva(1), Wilson Roberto Maluf(1), Maria das Graças Cardoso(2), Álvaro Carlos Gonçalves Neto(1), \\ Gabriel Mascarenhas Maciel( ${ }^{(1)}$, Daniela Aparecida Castro Nízio(3) e Vânia Aparecida Silva(4) \\ (1) Universidade Federal de Lavras (Ufla), Departamento de Agricultura, Caixa Postal 3.037, CEP $37200-000$ Lavras, MG. \\ E-mail: vanissesilva@yahoo.com, wrmaluf@ufla.br, alvarocgneto@gmail.com,gmmufla@hotmail.com, (2)Ufla, Departamento de Química. \\ E-mail: mcardoso@ufla.br ${ }^{(3)}$ Ufla, Departamento de Biologia. E-mail: danielanizio@hotmail.com ${ }^{(4)}$ Empresa de Pesquisa Agropecuária de \\ Minas Gerais, Caixa Postal 12, CEP 39525-000 Nova Porterinha, MG. E-mail: vaniaufv@yahoo.com.br
}

\begin{abstract}
Resumo - $\mathrm{O}$ objetivo deste trabalho foi comparar os graus de resistência à mosca-branca (Bemisia argentifolii) e ao ácaro-rajado (Tetranychus urticae) de híbridos de tomateiro resultantes do cruzamento entre linhagens com alto teor de zingibereno (ZGB) e linhagens com alto teor de acilaçúcar (AA), em contraste com as linhagens parentais e testemunhas comerciais. Foram avaliadas linhagens com altos teores de AA, linhagens com alto teor de ZGB, híbridos duplos heterozigotos ZGB+AA, híbridos heterozigotos para ZGB e híbridos heterozigotos para AA. Os acessos selvagens PI-127826 e LA-716 foram utilizados como testemunhas para alto teor de ZGB e AA, respectivamente, e os genótipos Débora Max e TOM-684 foram utilizados como testemunhas para baixo teor de ambos os aleloquímicos. Os genótipos foram submetidos ao teste de resistência à mosca-branca e ao teste de repelência ao ácaro. Os genótipos duplos heterozigotos apresentaram graus de resistência à mosca-branca superiores aos das testemunhas comerciais e inferiores aos das linhagens com alto ZGB ou com alto AA. Os genótipos duplos heterozigotos apresentaram maior repelência ao ácaro, em relação às testemunhas comerciais, e repelência semelhante à das linhagens com alto ZGB ou com alto AA. Não foi observado efeito sinérgico entre ZGB e AA nos genótipos duplos heterozigotos quanto à resistência à mosca-branca e repelência ao ácaro.
\end{abstract}

Termos para indexação: Bemisia argentifolii, Lycopersicon esculentum, Tetranychus urticae, aleloquímicos, resistência varietal, sesquiterpeno.

\section{Resistance mediated by alelochemicals of tomato genotypes to the silverleaf whitefly and to two-spotted spider mites}

\begin{abstract}
The objective of this work was to assess the degree of resistance to the whitefly (Bemisia argentifolii) and to the two-spotted spider mite (Tetranychus urticae) of tomato hybrids resulting from crosses between high-zingiberene (ZGB) lines and high-acylsugar (AS) lines, as compared to their parental lines and to commercial vouchers. High-AS lines, high-ZGB lines, double heterozygous (ZGB+AS) hybrids, hybrids heterozygous for ZGB and hybrids heterozygous for AS were tested. The wild accessions PI 127826 and LA-716 were used as high-ZGB and high-AS vouchers, respectively, while the genotypes Debora Max and TOM-684 were used as vouchers for low ZGB and AS. The genotypes were submitted to resistance tests to the whitefly and repellency to the spider mite. Double heterozygous (ZGB+AS) hybrids were more resistant to whiteflies than the commercial vouchers, but less resistant than the high-ZGB or the high-AS lines. Double heterozygous $(\mathrm{ZGB}+\mathrm{AS})$ hybrids were more repellent to the spider mite than the commercial vouchers, and their repellency to mites was similar to that of high-ZGB or high-AS lines. There was no synergistic effect between ZGB and AS, for both resistance to the whitefly and repellency to the two-spotted spider mite, in the double heterozygous (ZGB+AS) genotypes.
\end{abstract}

Index terms: Bemisia argentifolii, Lycopersicon esculentum, Tetranychus urticae, allelochemicals, varietal resistance, sesquiterpene.

\section{Introdução}

Apesar de o tomateiro (Lycopersicon esculentum Mill.) ser uma das mais importantes hortaliças cultivadas no mundo (Peralta et al., 2005), é uma planta que apresenta vários problemas fitossanitários (Suinaga et al., 2003). Entre os principais artrópodos-praga na cultura do tomateiro, destacam-se a mosca-branca e os ácaros. A mosca-branca pertence à família Aleyrodidae, e seu biótipo mais agressivo é Bemisia 
tabaci biótipo B ou, segundo Bellows et al. (1994), Bemisia argentifolii. A ocorrência de $B$. argentifolii em lavouras de tomate é bastante frequente e resulta, comumente, em danos diretos à produção, e em danos indiretos pela transmissão de fitoviroses causadas por geminivírus (Villas Bôas et al., 2002). Os ácaros do gênero Tetranychus, apesar de serem pragas de importância secundária, em condições de alta infestação podem provocar danos diretos às plantas e ocasionar o secamento das folhas, além de indução à maturação precoce (Flechtmann, 1989).

O controle químico desses artrópodos-praga é o principal método de manejo empregado. A utilização de defensivos, como única ou principal forma de manejo, pode acarretar danos ao ambiente e à saúde do trabalhador rural e do consumidor. Assim, o desenvolvimento de cultivares resistentes tem grande relevância.

Os programas de melhoramento do tomateiro para a obtenção de cultivares resistentes a artrópodos-praga, conduzidos no Brasil, têm adotado a estratégia de integração, em cultivares comerciais, de alelos de resistência presentes em materiais selvagens. A espécie Lycopersicon hirsutum Dunal - de acordo com Peralta et al. (2005) -, tem sido estudada quanto à presença de sesquiterpenos, especialmente o zingibereno (ZGB), que confere resistência a ácaros (Maluf et al., 2001; Gonçalves et al., 2006), à traça-do-tomateiro (Ecole et al., 2000; Azevedo et al., 2003) e à mosca-branca (Freitas et al., 2002). A espécie Lycopersicon pennellii (Correll) D'Arcy também tem sido utilizada em trabalhos sobre a resistência, em razão da presença de acilaçúcares (AA) (acilglicose e acilsacarose) em seus tecidos (Liedl et al., 1995; Freitas et al., 2002; Resende et al., 2008; Pereira et al., 2008). Em geral, a seleção de genótipos de tomateiro com alto teor desses aleloquímicos tem levado a respostas correlacionadas ao aumento da resistência a pragas. Esse procedimento tem apresentado maior eficiênca do que técnicas de seleção direta para resistência a pragas específicas.

$\mathrm{O}$ teor de ZGB apresenta herança monogênica, com dominância incompleta no sentido de menor teor, porém não se exclui a possibilidade de ocorrerem genes modificadores (Freitas et al., 2002). Por meio de modelos genéticos com uso da função de verossimilhança, Gonçalves et al. (2007) confirmaram que o alelo presente em $L$. pennelii, que confere alto teor de AA, é recessivo com grau de dominância incompleto. Apesar de se conhecerem as heranças genéticas dos teores de ZGB e AA, e de já terem sido documentadas as resistências a pragas em genótipos de tomateiro com alto teor de ZGB, ou de AA apenas, ainda não há relatos de híbridos que apresentem simultaneamente alto teor de ZGB e AA.

O objetivo deste trabalho foi comparar o grau de resistência à mosca-branca (B. argentifolii) e ao ácaro-rajado (T. urticae) de híbridos de tomateiro resultantes do cruzamento entre linhagens com alto teor de zingibereno e de acilaçúcares, em contraste com a resistência apresentada pelas linhagens parentais e por testemunhas comerciais.

\section{Material e Métodos}

Genótipos contrastantes quanto aos teores de zingibereno (ZGB) e acilaçúcares (AA) foram utilizados como parentais na formação de híbridos e como testemunhas comerciais nos bioensaios, para avaliação da resistência à mosca-branca (B. argentifolii) e da repelência ao ácaro-rajado (T. urticae) (Tabela 1). Os acessos selvagens PI-127826 e LA-716 foram utilizados como testemunhas quanto ao alto teor de ZGB e AA, respectivamente, e os genótipos Débora Max e TOM-684 foram utilizados como testemunhas quanto

Tabela 1. Teores médios de zingibereno e acilaçúcares, em genótipos de tomateiro utilizados como parentais na formação de híbridos e como testemunhas comerciais em bioensaios para a avaliação da resistência.

\begin{tabular}{|c|c|c|c|c|}
\hline \multirow[t]{2}{*}{ Genótipo } & \multicolumn{2}{|c|}{ Zingibereno } & \multicolumn{2}{|c|}{ Acilaçúcares } \\
\hline & $\begin{array}{c}\text { Teor médio } \\
\text { (absorbância) }\end{array}$ & $\begin{array}{c}\text { Teor relativo } \\
(\%)\end{array}$ & $\begin{array}{l}\text { Teor médio } \\
\left(\mathrm{nmol} \mathrm{cm} \mathrm{cm}^{-2}\right)\end{array}$ & $\begin{array}{c}\text { Teor relativo } \\
(\%)\end{array}$ \\
\hline Débora-Max e TOM-684 & 0,0816 & 100 & 7,03 & 100 \\
\hline TOM-688 e TOM-689 (homozigotos AA) & 0,1097 & 134 & 17,24 & 245 \\
\hline ZGB-703 e ZGB-704 (homozigotos ZGB) & 0,1882 & 231 & 12,84 & 183 \\
\hline Híbridos heterozigotos $\mathrm{ZGB}+\mathrm{AA}$ & 0,1514 & 186 & 16,46 & 234 \\
\hline Híbridos heterozigotos ZGB & 0,1518 & 186 & 12,89 & 183 \\
\hline Híbridos heterozigotos AA & 0,1111 & 136 & 18,00 & 256 \\
\hline Lycopersicon pennellii LA-716 & 0,1198 & 147 & 28,58 & 407 \\
\hline Lycopersicon hirsutum PI-127826 & 0,5883 & 721 & 12,13 & 173 \\
\hline
\end{tabular}


ao baixo teor de ambos os aleloquímicos. As linhagens TOM-688 e TOM-689 são linhagens pré-comerciais com alto teor de AA, resultantes do cruzamento interespecífico L. esculentum x L. pennellii LA-716, seguido por três retrocruzamentos com $L$. esculentum (Resende et al., 2008; Pereira et al., 2008). As linhagens ZGB-703 e ZGB-704 são linhagens com alto teor de ZGB, resultantes do cruzamento interespecífico L. esculentum x L.hirsutum PI-127826, seguido por dois retrocruzamentos com L. esculentum (Maluf et al., 2001; Freitas et al., 2002; Azevedo et al., 2003; Gonçalves et al., 2006).

As linhagens ZGB-703, ZGB-704, TOM-688 e TOM-689 foram cruzadas para obtenção de quatro genótipos duplos heterozigotos para AA e ZGB simultaneamente: $F_{1}$ (ZGB-703 x TOM-688), $\mathrm{F}_{1}\left(\mathrm{ZGB}-703 \times\right.$ TOM-689), $\mathrm{F}_{1}$ (ZGB-704 x TOM-688) e $F_{1}$ (ZGB-704 x TOM-689). As linhagens ZGB-703, ZGB-704, TOM-688 e TOM-689 também foram cruzadas com TOM-684, para obtenção de dois híbridos heterozigotos com elevado teor somente de ZGB - $F_{1}\left(Z G B-703 \times\right.$ TOM-684) e $F_{1}(Z G B-704 x$ TOM-684) -, e obtenção de dois híbridos heterozigotos com elevado teor somente de AA - $\mathrm{F}_{1}$ (TOM-688 x TOM-684) e $\mathrm{F}_{1}$ (TOM-689 x TOM-684).

Para a avaliação da resistência dos genótipos de tomateiro à mosca-branca, sementes de todos os genótipos foram semeadas em bandejas de poliestireno expandido, com substrato comercial Plantmax e casca de arroz carbonizada, na proporção 1:1; as mudas foram transplantadas, posteriormente, para vasos de $500 \mathrm{~mL}$, com o mesmo substrato. O delineamento utilizado foi o inteiramente casualizado, com 16 tratamentos e 6 repetições, no total de 96 plantas (1 planta por parcela). Sessenta dias após a semeadura, as plantas foram mantidas, por 48 horas, em estufa telada, infestada com população de $B$. argentifolii que havia sido mantida em plantas de tomate cultivar Santa Clara, em telados, no Departamento de Entomologoia da Universidade Federal de Lavras. Após essas 48 horas, as plantas foram retiradas da infestação para avaliação da ovoposição. A ovoposição foi avaliada na face abaxial tendo-se tomado a média de quatro folíolos da quarta folha a partir do ápice. Os ovos foram contados em uma área de $2 \mathrm{~cm}^{2}$, localizada no centro da folha e próxima ao pecíolo, com o auxílio de um microscópico estereoscópico binocular, com aumento de até 40 vezes. As folhas avaliadas foram previamente marcadas com uma fita adesiva, para contagem posterior do número de ninfas. Após a contagem do número de ovos, os vasos foram acondicionados em casa de vegetação livre de infestação e, após 20 dias, foi feita a avaliação do número de ninfas, com o cuidado de amostrar a mesma área avaliada para ovoposição. Para isso, a folha previamente marcada com a fita adesiva foi examinada com o auxílio de microscópio estereoscópico. A temperatura média e a umidade relativa do ar, do período de infestação das plantas até a contagem de ninfas, variaram, respectivamente, de 17 a $26,5^{\circ} \mathrm{C}$ e de 80 a $100 \%$.

Os ácaros utilizados foram provenientes de plantas altamente infestadas de tomate da cultivar Santa Clara e de feijão-vagem. Depois de coletados, os ácaros foram mantidos, para multiplicação, em novas plantas de tomate da cultivar Santa Clara e feijão-vagem, em vasos de $5 \mathrm{~L}$, em casa de vegetação da Estação Experimental de Hortaliças, da HortiAgro Sementes Ltda., na Fazenda Palmital, Ijaci, MG. A repelência ao ácaro T. urticae foi quantificada por meio do bioensaio proposto por Weston \& Snyder (1990). Os genótipos foram semeados e, após aproximadamente 30 dias, as mudas foram transplantadas para vasos de $500 \mathrm{~mL}$. $\mathrm{O}$ delineamento utilizado foi em blocos ao acaso, com 3 repetições, no total de 48 plantas. Aproximadamente 40 dias pós o transplante, foram retirados folíolos expandidos, de tamanhos semelhantes, do terço superior das plantas. Esses folíolos foram levados para uma câmara fria, conforme Weston \& Snyder (1990), com temperatura de $16 \pm 1^{\circ} \mathrm{C}$ e umidade relativa de $64 \pm 4 \%$. Um folíolo de cada um dos 16 genótipos foi fixado com uma tachinha metálica ( $9 \mathrm{~mm}$ de diâmetro), na região central da superfície foliar abaxial, em uma folha de papel branco, formato A4 $(210 \times 297 \mathrm{~mm})$, sobre uma placa de isopor. Os 16 folíolos foram distribuídos aleatoriamente sobre a placa de isopor. Foram coletados dez ácaros da criação, que foram transferidos para o centro de cada tachinha, com auxílio de um pincel fino. Após $20 \mathrm{~min}$, foi realizada a primeira medição das distâncias médias percorridas pelos ácaros (em $\mathrm{mm}$ ) sobre a superfície de cada folíolo, a partir do centro da tachinha. Decorridos outros $20 \mathrm{~min}$, foi feita a segunda medição de caminhada dos ácaros (tempo de avaliação $40 \mathrm{~min}$ ), e a terceira avaliação foi feita 20 min após a segunda (tempo de avaliação $60 \mathrm{~min}$ ). As menores distâncias percorridas pelos ácaros foram consideradas indicativas de maior grau de repelência 
(Weston \& Snyder, 1990). Foi considerada zero a distância percorrida pelos ácaros que permaneceram sobre a tachinha.

Com os resultados dos ensaios, procedeu-se à análise de variâncias, e as médias dos genótipos foram comparadas pelo teste de Scott-Knott, a 5\% de probabilidade, por meio do aplicativo estatístico Sisvar (Ferreira, 2000). Foram selecionados contrastes não ortogonais entre grupos de genótipos com teores diferentes de zingibereno e acilaçúcares (Tabela 2), para a caracterização de diferenças entre os graus de resistência a $B$. argentifolii e de repelência a $T$. urticae conferidos pelos dois aleloquímicos.

\section{Resultados e Discussão}

Foi possível verificar que tanto a ovoposição quanto a sobrevivência das ninfas de $B$. argentifolii foram afetadas pela presença dos aleloquímicos ZGB e AA, nos genótipos de tomateiro avaliados (Tabela 3). A presença de ao menos um dos aleloquímicos nos genótipos, em homozigose ou em heterozigose, foi determinante para a redução da ovoposição e da sobrevivência de ninfas, em relação às testemunhas Débora Max e TOM-684 (contrastes C1, C2, C3, C4 e C5, Tabela 3).

Os genótipos simultaneamente duplos heterozigotos para ZGB e AA [F $F_{1}(Z G B-703$ x TOM-688), $\mathrm{F}_{1}(\mathrm{ZGB}-703 \times \mathrm{TOM}-689), \mathrm{F}_{1}(\mathrm{ZGB}-704 \times$ xOM-688) e
$\mathrm{F}_{1}$ (ZGB-704 x TOM-689)] nãoapresentaram diferença significativa em relação aos híbridos heterozigotos somente para ZGB [F $($ ZGB-703 x TOM-684), $\mathrm{F}_{1}$ (ZGB-704 x TOM-684)] ou AA [F $\mathrm{F}_{1}(\mathrm{TOM}-688$ $\mathrm{x}$ TOM-684), $\mathrm{F}_{1}$ (TOM-689 x TOM-684)], seja quanto ao número médio de ovos, seja quanto à sobrevivência das ninfas de $B$. argentifolii (contrastes C6 e C7, Tabela 3). O fato de os genótipos duplos heterozigotos apresentarem o mesmo comportamento que os heterozigotos para ZGB ou AA indica que estes aleloquímicos atuam de forma semelhante quanto à resistência dos genótipos à mosca-branca, e que eles não apresentam efeito sinérgico. Esses resultados são contrastantes aos observados por Silva (2009), na traça-do-tomateiro Tuta absoluta, que constatou que a presença simultânea de ZGB e AA promoveu aumento no nível de resistência à traça, em relação à presença de somente ZGB ou de AA.

Com relação à ovoposição, ambos os aleloquímicos, quando em homozigose, apresentaram o mecanismo de resistência do tipo não preferência, e, entre as linhagens ricas em AA (TOM-688 e TOM-689) ou ricas em ZGB (ZGB-703 e ZGB-704), a linhagem ZGB-704 se destacou, apesar de não terem sido observadas diferenças significativas entre os tratamentos com os aleloquímicos em homozigose (contraste $\mathrm{C} 8$, Tabela 3). O ZGB mostrou-se significativamente mais eficiente do que o AA em reduzir o número de ninfas, apesar da pequena magnitude das diferenças observadas, o que

Tabela 2. Descrição dos contrastes de interesse, usados para comparações entre genótipos ou grupos de genótipos de tomateiro com diferentes teores de zingibereno e acilaçúcares.

\begin{tabular}{|c|c|c|}
\hline Contrastes & Contrastes estimados $^{(1)}$ & Descrição \\
\hline$\overline{\mathrm{C} 1}$ & {$[(\mathrm{~T} 1+\mathrm{T} 2) / 2-(\mathrm{T} 3+\mathrm{T} 4) / 2]$} & Testemunhas comerciais (baixo AA e baixo ZGB) x linhagens homozigotas com alto AA \\
\hline $\mathrm{C} 2$ & {$[(\mathrm{~T} 1+\mathrm{T} 2) / 2-(\mathrm{T} 5+\mathrm{T} 6) / 2]$} & Testemunhas comerciais (baixo AA e baixo ZGB) $\mathrm{x}$ linhagens homozigotas com alto ZGB \\
\hline $\mathrm{C} 3$ & {$[(\mathrm{~T} 1+\mathrm{T} 2) / 2-(\mathrm{T} 13+\mathrm{T} 14) / 2]$} & Testemunhas comerciais (baixo AA e baixo ZGB) $\mathrm{x}$ híbridos heterozigotos para AA \\
\hline $\mathrm{C} 4$ & {$[(\mathrm{~T} 1+\mathrm{T} 2) / 2-(\mathrm{T} 11+\mathrm{T} 12) / 2]$} & Testemunhas comerciais (baixo AA e baixo ZGB) $\mathrm{x}$ híbridos heterozigotos para ZGB \\
\hline $\mathrm{C} 5$ & {$[(\mathrm{~T} 1+\mathrm{T} 2) / 2-(\mathrm{T} 7+\mathrm{T} 8+\mathrm{T} 9+\mathrm{T} 10) / 4]$} & Testemunhas comerciais (baixo AA e baixo ZGB) x híbridos heterozigotos para AA e ZGB \\
\hline C6 & {$[(\mathrm{T} 13+\mathrm{T} 14) / 2-(\mathrm{T} 7+\mathrm{T} 8+\mathrm{T} 9+\mathrm{T} 10) / 4]$} & Híbridos heterozigotos para AA x híbridos heterozigotos para AA e ZGB \\
\hline C7 & {$[(\mathrm{T} 11+\mathrm{T} 12) / 2-(\mathrm{T} 7+\mathrm{T} 8+\mathrm{T} 9+\mathrm{T} 10) / 4]$} & Híbridos heterozigotos para ZGB x híbridos heterozigotos para AA e ZGB \\
\hline $\mathrm{C} 8$ & {$[(\mathrm{~T} 3+\mathrm{T} 4) / 2-(\mathrm{T} 5+\mathrm{T} 6) / 2]$} & Linhagens com alto AA (baixo ZGB) $x$ linhagens com alto ZGB (baixo AA) \\
\hline C9 & {$[(\mathrm{T} 3+\mathrm{T} 4) / 2-(\mathrm{T} 13+\mathrm{T} 14) / 2]$} & Linhagens com alto AA (baixo ZGB) $\mathrm{x}$ híbridos heterozigotos para AA \\
\hline $\mathrm{C} 10$ & {$[(\mathrm{~T} 3+\mathrm{T} 4) / 2-(\mathrm{T} 11+\mathrm{T} 12) / 2]$} & Linhagens com alto AA (baixo ZGB) $\mathrm{x}$ híbridos heterozigotos para ZGB \\
\hline C11 & {$[(\mathrm{T} 3+\mathrm{T} 4) / 2-(\mathrm{T} 7+\mathrm{T} 8+\mathrm{T} 9+\mathrm{T} 10) / 4]$} & Linhagens com alto AA (baixo $\mathrm{ZGB}$ ) $\mathrm{x}$ híbridos heterozigotos para $\mathrm{ZGB}+\mathrm{AA}$ \\
\hline $\mathrm{C} 12$ & {$[(\mathrm{~T} 5+\mathrm{T} 6) / 2-(\mathrm{T} 13+\mathrm{T} 14) / 2]$} & Linhagens com alto ZGB (baixo AA) $\mathrm{x}$ híbridos heterozigotos para AA \\
\hline $\mathrm{C} 13$ & {$[(\mathrm{~T} 5+\mathrm{T} 6) / 2-(\mathrm{T} 11+\mathrm{T} 12) / 2]$} & Linhagens com alto ZGB (baixo AA) $\mathrm{x}$ híbridos heterozigotos para ZGB \\
\hline $\mathrm{C} 14$ & $(\mathrm{~T} 5+\mathrm{T} 6) / 2-(\mathrm{T} 7+\mathrm{T} 8+\mathrm{T} 9+\mathrm{T} 10) / 4]$ & Linhagens com alto $\mathrm{ZGB}$ (baixo $\mathrm{AA}$ ) $\mathrm{x}$ híbridos heterozigotos para $\mathrm{ZGB}+\mathrm{AA}$ \\
\hline $\mathrm{C} 15$ & {$[(\mathrm{~T} 3+\mathrm{T} 4+\mathrm{T} 5+\mathrm{T} 6) / 4-(\mathrm{T} 7+\mathrm{T} 8+\mathrm{T} 9+\mathrm{T} 10+\mathrm{T} 11+\mathrm{T} 12+\mathrm{T} 13+\mathrm{T} 14) / 8]$} & $\begin{array}{l}\text { Linhagens com alto AA (baixo ZGB) e com alto ZGB (baixo AA) } \mathrm{x} \text { híbridos heterozigotos para } \\
\mathrm{AA} \text {, para ZGB, e para ZGB + AA }\end{array}$ \\
\hline $\mathrm{C} 16$ & {$[(\mathrm{~T} 3+\mathrm{T} 4+\mathrm{T} 5+\mathrm{T} 6) / 4-(\mathrm{T} 15+\mathrm{T} 16) / 2]$} & $\begin{array}{l}\text { Linhagens com alto AA (baixo ZGB) e com alto ZGB (baixo AA) } x \text { acessos selvagens PI } \\
127826 \text { e LA-716 }\end{array}$ \\
\hline $\mathrm{C} 17$ & {$[(\mathrm{~T} 7+\mathrm{T} 8+\mathrm{T} 9+\mathrm{T} 10+\mathrm{T} 11+\mathrm{T} 12+\mathrm{T} 13+\mathrm{T} 14) / 8-(\mathrm{T} 15+\mathrm{T} 16) / 2]$} & $\begin{array}{l}\text { Híbridos heterozigotos para AA, para ZGB, e para ZGB + AA x acessos selvagens PI } 127826 \text { e } \\
\text { LA-716 }\end{array}$ \\
\hline
\end{tabular}

(1)T1, Debora Max; T2, TOM-684; T3, TOM-688; T4, TOM-689; T5, ZGB-703; T6, ZGB-704; T7, F1(ZGB-703 x TOM-688); T8, F1(ZGB-703 x TOM-689); T9, F1(ZGB-704 x TOM-688); T10, F1(ZGB-704 x TOM-689); T11, F1(ZGB-703 x TOM-684); T12, F1(ZGB-704 x TOM-684); T13, F1(TOM-688 x TOM-684); T14, F1(TOM-689 x TOM-684); T15, PI-127826; T16, LA-716. 
indica que o ZGB pode conferir maior grau de antibiose do que o AA. De maneira geral, as linhagens com alto teor de ZGB ou de AA apresentaram maior resistência à mosca-branca do que os genótipos heterozigotos somente para AA $\left[\mathrm{F}_{1}\left(\mathrm{TOM}-688\right.\right.$ x TOM-684), $\mathrm{F}_{1}$ (TOM-689 x TOM-684)] ou heterozigotos somente para ZGB [F F $_{1}$ ZGB-703 x TOM-684), F $_{1}$ (ZGB-704 x TOM-684)] e simultaneamente duplos heterozigotos para ZGB e AA [F F $_{1}$ ZGB-703 x TOM-688), $\mathrm{F}_{1}$ (ZGB-703 x TOM-689), $F_{1}$ (ZGB-704 x TOM-688), $F_{1}$ (ZGB-704 x TOM-689)] (contrastes C12, C13 e C14, Tabela 3). Para o aleloquímico $\mathrm{AA}$, essa superioridade dos homozigotos foi mais aparente no número de ninfas (contrastes C9, C10 e C11, Tabela 3), enquanto para o ZGB, a maior resistência dos genótipos homozigotos ficou evidente tanto na ovoposição quanto no número de ninfas, o que indica que a presença de ZGB em homozigose nos genótipos de tomateiro pode ser mais eficiente do que o AA em homozigose, em conferir resistência à mosca-branca. A efetividade do ZGB em conferir resistência à $B$. argentifolii foi comprovada inicialmente por Freitas et al. (2002), que verificaram que os genótipos selecionados para alto teor de ZGB apresentaram maiores níveis de resistência à mosca-branca do que o tomateiro comercial com baixo teor de ZGB.

Embora, no presente trabalho, os genótipos duplos heterozigotos simultaneamente para $\mathrm{ZGB}$ e AA [ $\mathrm{F}_{1}\left(\mathrm{ZGB}-703 \times\right.$ TOM-688), $\mathrm{F}_{1}$ (ZGB-703 x TOM-689), $\mathrm{F}_{1}$ (ZGB-704 x TOM-688), $\mathrm{F}_{1}$ (ZGB-704 x TOM-689)] não tenham se mostrado mais resistentes à mosca-branca do que os genótipos heterozigotos somente para ZGB [F ${ }_{1}$ (ZGB-703 x TOM-684), $\mathrm{F}_{1}$ (ZGB-704 x TOM-684)] ou somente para AA $\left[\mathrm{F}_{1}(\mathrm{TOM}-688 \times \mathrm{TOM}-684)\right.$, $\mathrm{F}_{1}$ (TOM-689 x TOM-684)], a obtenção destes

Tabela 3. Número médio de ovos e ninfas por $2 \mathrm{~cm}^{2}$ de área foliar de genótipos de tomateiro, medido, respectivamente, aos 2 e 20 dias após a infestação da mosca-branca Bemisia argentifolii, e estimativas de contrastes de interesse ${ }^{(1)}$.

\begin{tabular}{|c|c|c|}
\hline Genótipos & Média de ovos & Média de ninfas \\
\hline T1, Débora Max & $104,83 \mathrm{D}$ & $85,00 \mathrm{D}$ \\
\hline T2, TOM-684 & $96,16 \mathrm{D}$ & $70,66 \mathrm{D}$ \\
\hline T3, TOM-688 & $51,33 \mathrm{C}$ & $46,33 \mathrm{C}$ \\
\hline T5, ZGB-703 & $41,83 \mathrm{C}$ & $33,16 \mathrm{~B}$ \\
\hline T6, ZGB-704 & $31,33 \mathrm{~B}$ & $25,33 \mathrm{~B}$ \\
\hline T7, $\mathrm{F}_{1}(\mathrm{ZGB}-703 \times \mathrm{TOM}-688)$ & $56,83 \mathrm{C}$ & $51,50 \mathrm{C}$ \\
\hline T8, $F_{1}(Z G B-703 \times$ TOM-689) & $56,00 \mathrm{C}$ & $53,83 \mathrm{C}$ \\
\hline T10, $\mathrm{F}_{1}(\mathrm{ZGB}-704 \times \mathrm{TOM}-689)$ & $49,83 \mathrm{C}$ & $46,33 \mathrm{C}$ \\
\hline T11, $F_{1}(Z G B-703 \times$ TOM-684) & $52,33 \mathrm{C}$ & $47,33 \mathrm{C}$ \\
\hline $\mathrm{T} 12, \mathrm{~F}_{1}(\mathrm{ZGB}-704 \times \mathrm{TOM}-684)$ & $61,50 \mathrm{C}$ & $57,16 \mathrm{C}$ \\
\hline $\mathrm{T} 13, \mathrm{~F}_{1}(\mathrm{TOM}-688 \times \mathrm{TOM}-684)$ & $62,16 \mathrm{C}$ & $54,66 \mathrm{C}$ \\
\hline T14, F (TOM-689 x TOM-684) & $57,83 \mathrm{C}$ & $53,83 \mathrm{C}$ \\
\hline T15, PI-127826 = Lycopersicon hirsutum & $0,16 \mathrm{~A}$ & $0,00 \mathrm{~A}$ \\
\hline T16, LA-716 = Lycopersicon pennellii & $0,33 \mathrm{~A}$ & $0,33 \mathrm{~A}$ \\
\hline \multicolumn{3}{|l|}{ Contrastes de interesse } \\
\hline $\mathrm{C} 2=(\mathrm{T} 1+\mathrm{T} 2) / 2-(\mathrm{T} 5+\mathrm{T} 6) / 2$ & $63,91 * *$ & $48,58 * *$ \\
\hline $\mathrm{C} 3=(\mathrm{T} 1+\mathrm{T} 2) / 2-(\mathrm{T} 13+\mathrm{T} 14) / 2$ & $40,50 * *$ & $23,58 * *$ \\
\hline $\mathrm{C} 4=(\mathrm{T} 1+\mathrm{T} 2) / 2-(\mathrm{T} 11+\mathrm{T} 12) / 2$ & $43,58 * *$ & $25,58 * *$ \\
\hline $\mathrm{C} 5=(\mathrm{T} 1+\mathrm{T} 2) / 2-(\mathrm{T} 7+\mathrm{T} 8+\mathrm{T} 9+\mathrm{T} 10) / 4$ & $46,50 * *$ & $27,62 * *$ \\
\hline $\mathrm{C} 6=(\mathrm{T} 13+\mathrm{T} 14) / 2-(\mathrm{T} 7+\mathrm{T} 8+\mathrm{T} 9+\mathrm{T} 10) / 4$ & $6,00^{\mathrm{ns}}$ & $4,04^{\mathrm{ns}}$ \\
\hline $\mathrm{C} 7=(\mathrm{T} 11+\mathrm{T} 12) / 2-(\mathrm{T} 7+\mathrm{T} 8+\mathrm{T} 9+\mathrm{T} 10) / 4$ & $2,91^{\mathrm{ns}}$ & $2,04^{\mathrm{ns}}$ \\
\hline $\mathrm{C} 8=(\mathrm{T} 3+\mathrm{T} 4) / 2-(\mathrm{T} 5+\mathrm{T} 6) / 2$ & $9,91^{\mathrm{ns}}$ & $10,66^{*}$ \\
\hline $\mathrm{C} 9=(\mathrm{T} 3+\mathrm{T} 4) / 2-(\mathrm{T} 13+\mathrm{T} 14) / 2$ & $-13,50 *$ & $-14,33 * *$ \\
\hline $\mathrm{C} 10=(\mathrm{T} 3+\mathrm{T} 4) / 2-(\mathrm{T} 11+\mathrm{T} 12) / 2$ & $-10,41^{\mathrm{ns}}$ & $-12,33^{*}$ \\
\hline $\mathrm{C} 11=(\mathrm{T} 3+\mathrm{T} 4) / 2-(\mathrm{T} 7+\mathrm{T} 8+\mathrm{T} 9+\mathrm{T} 10) / 4$ & $-7,50^{\mathrm{ns}}$ & $-10,29 *$ \\
\hline $\mathrm{C} 12=(\mathrm{T} 5+\mathrm{T} 6) / 2-(\mathrm{T} 13+\mathrm{T} 14) / 2$ & $-23,41 * *$ & $-25,00 * *$ \\
\hline $\mathrm{C} 13=(\mathrm{T} 5+\mathrm{T} 6) / 2-(\mathrm{T} 11+\mathrm{T} 12) / 2$ & $-20,33 * *$ & $-23,00 * *$ \\
\hline $\mathrm{C} 14=(\mathrm{T} 5+\mathrm{T} 6) / 2-(\mathrm{T} 7+\mathrm{T} 8+\mathrm{T} 9+\mathrm{T} 10) / 4$ & $-17,41 * *$ & $-20,95 * *$ \\
\hline $\mathrm{C} 15=(\mathrm{T} 3+\mathrm{T} 4+\mathrm{T} 5+\mathrm{T} 6) / 4-(\mathrm{T} 7+\mathrm{T} 8+\mathrm{T} 9+\mathrm{T} 10+\mathrm{T} 11+\mathrm{T} 12+\mathrm{T} 13+\mathrm{T} 14) / 8$ & $-14,68 * *$ & $-17,14 * *$ \\
\hline $\mathrm{C} 16=(\mathrm{T} 3+\mathrm{T} 4+\mathrm{T} 5+\mathrm{T} 6) / 4-(\mathrm{T} 15+\mathrm{T} 16) / 2$ & $41,29 * *$ & $34,41 * *$ \\
\hline $\mathrm{C} 17=(\mathrm{T} 7+\mathrm{T} 8+\mathrm{T} 9+\mathrm{T} 10+\mathrm{T} 11+\mathrm{T} 12+\mathrm{T} 13+\mathrm{T} 14) / 8-(\mathrm{T} 15+\mathrm{T} 1) / 2$ & $55,97 * *$ & $51,56 * *$ \\
\hline $\mathrm{CV}(\%)$ & $29,28 \%$ & $28,71 \%$ \\
\hline
\end{tabular}

${ }^{(1)}$ Médias seguidas por letras iguais, nas colunas, não diferem entre si pelo teste de Scott-Knott a $5 \%$ de probabilidade. ${ }^{\text {ns }}$ Não significativo. * e **Significativo pelo teste $\mathrm{F}$ a 5 e $1 \%$ de probabilidade, respectivamente. 
genótipos duplos heterozigotos poderia acarretar maior resistência a outros artrópodos-praga do tomateiro, e aumentar o espectro de ação do efeito isolado desses aleloquímicos. Genótipos ricos simultaneamente em ZGB e AA também poderiam atuar como barreira mais efetiva contra biótipos de artrópodos-praga que viessem a quebrar a resistência mediada por apenas um dos aleloquímicos isoladamente. Os possíveis efeitos de altos teores de ZGB ou AA ou de ambos sobre os inimigos naturais de pragas do tomateiro não podem ser previstos a priori; contudo, em uma situação relatada de resistência à traça Tuta absoluta, em tomateiro rico em outro grupo de aleloquímicos (metil-cetonas), a mesma substância que confere resistência à traça (2-tridecanona) atuou de forma negativa sobre o parasitismo de seus ovos por Trichogramma pretiosum (Gonçalves-Gervásio et al., 2000)
Os genótipos testados apresentaram diferenças significativas (Tabela 4) quanto à repelência ao ácaro. Os genótipos com alto teor de AA somente, com alto teor de ZGB somente, aqueles simultaneamente com alto teor em ZGB e AA, as linhagens com alto teor em ZGB e aquelas com alto teor em AA apresentaram distâncias de caminhada dos ácaros sobre os folíolos significativamente menores, em todos os tempos de avaliação, quando comparados com as testemunhas comerciais com baixo teor de ZGB e AA (contrastes C1, C2, C3, C4, C5, Tabela 4). Esse resultado evidencia a eficiência de ambos os aleloquímicos em causar repelência ao ácaro $T$. urticae. No entanto, mesmo com o alto grau de repelência encontrado, nenhum dos genótipos avaliados foi tão repelente ao ácaro T. urticae quanto os acessos selvagens PI-127826 e LA-716 (contrastes C16, C17, Tabela 4),

Tabela 4. Distâncias médias percorridas pelos ácaros Tetranychus urticae na superfície dos folíolos de genótipos de tomateiro em diferentes tempos de avaliação, e estimativas de contrastes de interesse ${ }^{(1)}$.

\begin{tabular}{|c|c|c|c|}
\hline \multirow[t]{2}{*}{ Tratamentos } & \multicolumn{3}{|c|}{ Distância (mm) } \\
\hline & $20 \mathrm{~min}$ & $40 \mathrm{~min}$ & $60 \mathrm{~min}$ \\
\hline T1, Débora Max & $19,28 \mathrm{D}$ & $18,88 \mathrm{E}$ & $22,60 \mathrm{~F}$ \\
\hline T2, TOM-684 & $21,85 \mathrm{E}$ & $21,44 \mathrm{~F}$ & $18,66 \mathrm{E}$ \\
\hline T3, TOM-688 & $9,03 \mathrm{~B}$ & $8,94 \mathrm{~B}$ & $11,43 \mathrm{C}$ \\
\hline T4, TOM-689 & $11,33 \mathrm{C}$ & $13,06 \mathrm{D}$ & $13,94 \mathrm{D}$ \\
\hline T5, ZGB-703 & $12,26 \mathrm{C}$ & $12,09 \mathrm{D}$ & $13,18 \mathrm{D}$ \\
\hline T6, ZGB-704 & $11,46 \mathrm{C}$ & $9,51 \mathrm{~B}$ & $11,17 \mathrm{C}$ \\
\hline T7, $F_{1}(Z G B-703 \times$ TOM-688) & $10,55 \mathrm{C}$ & $10,85 \mathrm{C}$ & $11,70 \mathrm{C}$ \\
\hline T8, F 1 (ZGB-703 x TOM-689) & $11,76 \mathrm{C}$ & $8,03 \mathrm{~B}$ & $9,34 \mathrm{~B}$ \\
\hline T9, $\mathrm{F}_{1}(\mathrm{ZGB}-704 \times \mathrm{TOM}-688)$ & $9,56 \mathrm{~B}$ & $9,21 \mathrm{~B}$ & $9,34 \mathrm{~B}$ \\
\hline T10, $\mathrm{F}_{1}(\mathrm{ZGB}-704$ x TOM-689) & $11,04 \mathrm{C}$ & $10,42 \mathrm{C}$ & $9,60 \mathrm{~B}$ \\
\hline $\mathrm{T} 11, \mathrm{~F}_{1}(\mathrm{ZGB}-703 \times \mathrm{TOM}-684)$ & $9,14 \mathrm{~B}$ & $9,13 \mathrm{~B}$ & $9,13 \mathrm{~B}$ \\
\hline $\mathrm{T} 12, \mathrm{~F}_{1}(\mathrm{ZGB}-704 \times \mathrm{TOM}-684)$ & $9,28 \mathrm{~B}$ & $8,85 \mathrm{~B}$ & $8,75 \mathrm{~B}$ \\
\hline T13, $\mathrm{F}_{1}(\mathrm{TOM}-688 \times \mathrm{TOM}-684)$ & $10,69 \mathrm{C}$ & $10,73 \mathrm{C}$ & $12,05 \mathrm{C}$ \\
\hline T14, $\mathrm{F}_{1}(\mathrm{TOM}-689 \times \mathrm{TOM}-684)$ & $11,79 \mathrm{C}$ & $11,90 \mathrm{D}$ & $11,37 \mathrm{C}$ \\
\hline T15, PI-127826 =Lycopersicon hirsutum & $2,22 \mathrm{~A}$ & $2,21 \mathrm{~A}$ & $2,24 \mathrm{~A}$ \\
\hline T16, LA-716 = Lycopersicon pennellii & $1,05 \mathrm{~A}$ & $1,17 \mathrm{~A}$ & $1,46 \mathrm{~A}$ \\
\hline \multicolumn{4}{|l|}{ Contrastes de interesse } \\
\hline $\mathrm{C} 1=(\mathrm{T} 1+\mathrm{T} 2) / 2-(\mathrm{T} 3+\mathrm{T} 4) / 2$ & $10,38 * *$ & $9,16^{* *}$ & $7,94 * *$ \\
\hline $\mathrm{C} 2=(\mathrm{T} 1+\mathrm{T} 2) / 2-(\mathrm{T} 5+\mathrm{T} 6) / 2$ & $8,70^{* *}$ & $9,36^{* *}$ & $8,45^{* *}$ \\
\hline $\mathrm{C} 3=(\mathrm{T} 1+\mathrm{T} 2) / 2-(\mathrm{T} 13+\mathrm{T} 14) / 2$ & $9,32 * *$ & $8,84 * *$ & $8,92 * *$ \\
\hline $\mathrm{C} 4=(\mathrm{T} 1+\mathrm{T} 2) / 2-(\mathrm{T} 11+\mathrm{T} 12) / 2$ & $11,35^{* *}$ & $11,17 * *$ & $11,69 * *$ \\
\hline $\mathrm{C} 5=(\mathrm{T} 1+\mathrm{T} 2) / 2-(\mathrm{T} 7+\mathrm{T} 8+\mathrm{T} 9+\mathrm{T} 10) / 4$ & $9,83 * *$ & $10,55^{* *}$ & $10,63 * *$ \\
\hline $\mathrm{C} 6=(\mathrm{T} 13+\mathrm{T} 14) / 2-(\mathrm{T} 7+\mathrm{T} 8+\mathrm{T} 9+\mathrm{T} 10) / 4$ & $0,51^{\mathrm{ns}}$ & $1,71 * *$ & $1,71 * *$ \\
\hline $\mathrm{C} 7=(\mathrm{T} 11+\mathrm{T} 12) / 2-(\mathrm{T} 7+\mathrm{T} 8+\mathrm{T} 9+\mathrm{T} 10) / 4$ & $-1,51 *$ & $-0,61^{\mathrm{ns}}$ & $-1,05^{*}$ \\
\hline $\mathrm{C} 8=(\mathrm{T} 3+\mathrm{T} 4) / 2-(\mathrm{T} 5+\mathrm{T} 6) / 2$ & $-1,68^{*}$ & $0,20^{\text {ns }}$ & $0,51^{\mathrm{ns}}$ \\
\hline $\mathrm{C} 9=(\mathrm{T} 3+\mathrm{T} 4) / 2-(\mathrm{T} 13+\mathrm{T} 14) / 2$ & $-1,06^{\mathrm{ns}}$ & $-0,31^{\mathrm{ns}}$ & $0,97^{\mathrm{ns}}$ \\
\hline $\mathrm{C} 10=(\mathrm{T} 3+\mathrm{T} 4) / 2-(\mathrm{T} 11+\mathrm{T} 12) / 2$ & $0,97^{\mathrm{ns}}$ & $2,01 * *$ & $3,74 * *$ \\
\hline $\mathrm{C} 11=(\mathrm{T} 3+\mathrm{T} 4) / 2-(\mathrm{T} 7+\mathrm{T} 8+\mathrm{T} 9+\mathrm{T} 10) / 4$ & $-0,54^{\mathrm{ns}}$ & $1,39 * *$ & $2,69 * *$ \\
\hline $\mathrm{C} 12=(\mathrm{T} 5+\mathrm{T} 6) / 2-(\mathrm{T} 13+\mathrm{T} 14) / 2$ & $0,62^{\text {ns }}$ & $-0,51^{\mathrm{ns}}$ & $0,46^{\mathrm{ns}}$ \\
\hline $\mathrm{C} 13=(\mathrm{T} 5+\mathrm{T} 6) / 2-(\mathrm{T} 11+\mathrm{T} 12) / 2$ & $2,65 * *$ & $1,81^{* *}$ & $3,23 * *$ \\
\hline $\mathrm{C} 14=(\mathrm{T} 5+\mathrm{T} 6) / 2-(\mathrm{T} 7+\mathrm{T} 8+\mathrm{T} 9+\mathrm{T} 10) / 4$ & $1,13^{\mathrm{ns}}$ & $1,19 * *$ & $2,18 * *$ \\
\hline $\mathrm{C} 15=(\mathrm{T} 3+\mathrm{T} 4+\mathrm{T} 5+\mathrm{T} 6) / 4-(\mathrm{T} 7+\mathrm{T} 8+\mathrm{T} 9+\mathrm{T} 10+\mathrm{T} 11+\mathrm{T} 12+\mathrm{T} 13+\mathrm{T} 14) / 8$ & $0,54^{\mathrm{ns}}$ & $1,02 * *$ & $2,27 * *$ \\
\hline $\mathrm{C} 16=(\mathrm{T} 3+\mathrm{T} 4+\mathrm{T} 5+\mathrm{T} 6) / 4-(\mathrm{T} 15+\mathrm{T} 16) / 2$ & $9,38 * *$ & $9,21 * *$ & $10,58 * *$ \\
\hline $\mathrm{C} 17=(\mathrm{T} 7+\mathrm{T} 8+\mathrm{T} 9+\mathrm{T} 10+\mathrm{T} 11+\mathrm{T} 12+\mathrm{T} 13+\mathrm{T} 14) / 8-(\mathrm{T} 15+\mathrm{T} 1) / 2$ & $8,84 * *$ & $8,18 * *$ & $8,31 * *$ \\
\hline CV (\%) & 20,49 & 13,20 & 15,06 \\
\hline
\end{tabular}

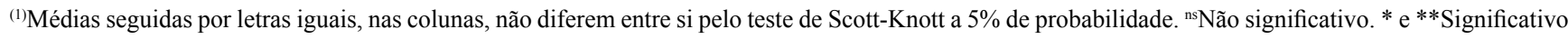
pelo teste $\mathrm{F}$ a 5 e $1 \%$ de probabilidade, respectivamente. 
os quais, adicionalmente, podem possuir outros fatores arrestantes ao caminhamento do ácaro, não presentes nas linhagens de tomateiro melhoradas. No menor tempo de avaliação (20 min), os genótipos simultaneamente duplos heterozigotos, quanto à $\mathrm{ZGB}$ e AA $\left[\mathrm{F}_{1}\right.$ (ZGB-703 x TOM-688), $F_{1}\left(Z G B-703 \times\right.$ TOM-689), $F_{1}$ (ZGB-704 x TOM-688), $\mathrm{F}_{1}$ (ZGB-704 x TOM-689)] mostraramse semelhantes aos genótipos heterozigotos somente quanto à $\mathrm{AA}\left[\mathrm{F}_{1}\left(\mathrm{TOM}-688 \times\right.\right.$ TOM-684), $\mathrm{F}_{1}$ (TOM-689 $\mathrm{x}$ TOM-684)], às linhagens com alto teor de AA (TOM-688 e TOM-689) e às linhagens com alto teor de ZGB (ZGB-703 e ZGB-704); porém, nos tempos de avaliações seguintes (40 e $60 \mathrm{~min}$ ), os genótipos duplos heterozigotos apresentaram menor distância de caminhada do ácaro T. urticae (contrastes C6, C11 e $\mathrm{C} 14$, Tabela 4). Os genótipos heterozigotos somente para ZGB $\left[\mathrm{F}_{1}\right.$ (ZGB-703 x TOM-684), $\mathrm{F}_{1}$ (ZGB-704 $\mathrm{x}$ TOM-684)] foram mais repelentes aos ácaros em comparação aos simultaneamente duplos heterozigotos para ZGB e AA (contraste C7, Tabela 4).

De maneira geral, na comparação entre linhagens com alto teor de AA (TOM-688 e TOM-689) com os híbridos heterozigotos somente para AA [ $\mathrm{F}_{1}$ (TOM-688 x TOM-684), $F_{1}$ (TOM-689 x TOM-684)] ou com as linhagens com alto teor de ZGB (ZGB-703 e ZGB-704), não se observaram diferenças significativas quanto à repelência ao ácaro $T$. urticae (contrastes C8 e C9, Tabela 4). Este resultado é indicação de que a ligeira superioridade quanto à repelência ao ácaro T. urticae, nos genótipos duplos heterozigotos, não pode ser atribuída à sinergia entre ZGB e AA, contrariamente ao que foi demonstrado nos testes de resistência à traça-do-tomateiro $T$. absoluta, realizados com os mesmos genótipos (Silva, 2009). Tais discrepâncias talvez reflitam apenas diferenças no background genotípico das linhagens empregadas na obtenção destes híbridos duplamente heterozigotos. Principalmente a partir do segundo tempo de avaliação (40 min), os híbridos heterozigotos somente para ZGB [F F $_{1}$ ZGB-703 x TOM-684), F (ZGB-704 x TOM-684)] apresentaram menor distância de caminhada do ácaro T. urticae do que as linhagens com alto teor de ZGB (ZGB-703 e ZGB-704), ou de linhagens com alto teor de AA (TOM-688 e TOM-689) (contrastes C10 e C13, Tabela 4)

Os híbridos heterozigotos somente para AA $\left(\left[\mathrm{F}_{1}\left(\mathrm{TOM}-688 \quad \mathrm{x} \quad\right.\right.\right.$ TOM-684), $\quad \mathrm{F}_{1} \quad$ (TOM-689 $\mathrm{x}$ TOM-684)] não apresentaram diferenças significativas quanto à repelência ao ácaro $T$. urticae em relação às linhagens homozigotas com alto teor de ZGB (ZGB-703 e ZGB-704), nos três tempos avaliados (contraste $\mathrm{C} 12$, Tabela 4). Com o aumento do tempo de exposição, o grupo formado por todos os híbridos foi mais repelente ao ácaro T. urticae do que o grupo formado pelas linhagens com alto teor em ZGB e as linhagens com alto teor em AA (contraste C15, Tabela 4). O fato de os híbridos apresentarem maior repelência ao ácaro T. urticae do que as linhagens homozigotas para ZGB e AA é indicação de que, com apenas uma linhagem com alto teor de ZGB, torna-se possível a obtenção de combinações híbridas com resistência ao ácaro T. urticae, e que não há necessidade, portanto, da utilização de dois parentais com alto teor de ZGB, o que tornaria restrito o número de combinações híbridas. Silva (2009) verificou que os dois aleloquímicos, quando combinados em híbridos heterozigotos, mostraram efeito sinérgico no aumento da resistência à traça-do-tomateiro. Este efeito sinérgico de AA e ZGB não foi observado no presente ensaio, no que se refere à repelência ao ácaro $T$. urticae.

\section{Conclusões}

1. Os genótipos duplos heterozigotos apresentam graus de resistência à mosca-branca Bemisia argentifolii superiores aos das testemunhas comerciais.

2. Os genótipos duplos heterozigotos apresentam graus de resistência à mosca-branca inferiores aos das linhagens com alto teor de zingebereno e aos daquelas com alto teor de acilaçúcares e, portanto, não evidenciam efeito sinérgico entre esses aleloquímicos.

3. Os genótipos duplos heterozigotos apresentam maior repelência ao ácaro Tetranychus urticae, em comparação às testemunhas comercias.

4. Não há efeito sinérgico entre zingebereno e acilaçúcares nos genótipos duplos heterozigotos também no que tange à repelência ao ácaro $T$. urticae.

\section{Agradecimentos}

Ao Conselho Nacional de Desenvolvimento Científico e Tecnológico, à Coordenação de Aperfeiçoamento de Pessoal de Nível Superior, à Fundação de Amparo à Pesquisa do Estado de Minas Gerais, ao Ministério da Ciência e Tecnologia, à Universidade Federal de Lavras, à HortiAgro Sementes Ltda., à Fundação para o Desenvolvimento Científico e Cultural e à Fundação de Apoio ao Ensino, Pesquisa e Extensão. 


\section{Referências}

AZEVEDO, S.M. de; FARIA, M.V.; MALUF, W.R.; OLIVEIRA, A.C.B.; FREITAS, J.A. de. Zingiberene-mediated resistance to the South American tomato pinworm derived from Lycopersicon hirsutum var. hirsutum. Euphytica, v.134, p.347-351, 2003.

BELLOWS, T.S.; PERRING, T.M.; GILL, R.J.; HEADRICK, D.H. Description of a species of Bemisia (Homoptera: Aleyrodidae). Annals of the Entomological Society of America, v.87, p.195-206, 1994.

ECOLE, C.C.; PICANÇO, M.; MOREIRA, M.D.; MAGALHÃES, S.T.V. Componentes químicos associados à resistência de Lycopersicon hirsutum f. typicum a Tuta absoluta (Meyrick) (Lepidoptera: Gelechiidae). Anais da Sociedade Entomológica do Brasil, v.29, p.327-337, 2000.

FERREIRA, D.F. Análises estatísticas por meio do Sisvar para Windows 4.0. In: REUNIÃO ANUAL DA REGIÃO BRASILEIRA DA SOCIEDADE INTERNACIONAL DE BIOMETRIA, 45. 2000, São Carlos. Anais. São Carlos: Universidade Federal de São Carlos, 2000. p.255-258.

FLECHTMANN, C.H.W. Ácaros de importância agrícola. São Paulo: Nobel, 1989. 189p.

FREITAS, J.A.; MALUF, W.R.; CARDOSO, M.D.; GOMES, L.A.A.; BEARZOTTI, E. Inheritance of foliar zingiberene contents and their relationship to trichome densities and whitefly resistance in tomatoes. Euphytica, v.127, p.275-287, 2002.

GONÇALVES, L.D.; MALUF, W.R.; CARDOSO, M. das G.; GOMES, L.A.A.; NASCIMENTO, I.R. do. Herança de acilaçúcares em genótipos de tomateiro provenientes de cruzamento interespecífico. Pesquisa Agropecuária Brasileira, v.42, p.699-705, 2007.

GONÇALVES, L.D.; MALUF, W.R.; CARDOSO, M. das G.; RESENDE, J.T.V. de; CASTRO, E.M. de; SANTOS, N.M.; NASCIMENTO, I.R. do; FARIA, M.V. Relação entre zingibereno, tricomas foliares e repelência de tomateiros a Tetranychus evansi. Pesquisa Agropecuária Brasileira, v.41, p.267-273, 2006.

GONÇALVES-GERVÁSIO, R.C.R.; CIOCIOLA, A.I.; SANTA-CECÍLIA, L.V.C.; MALUF, W.R. Parasitismo de ovos de Tuta absoluta por Trichogramma pretiosum em diferentes genótipos de tomateiro. Pesquisa Agropecuária Brasileira, v.35, p-1269-1274, 2000.

LIEDL, B.E.; LAWSON, D.M.; WHITE, K.K.; SIIAPIHO, J.A.; COHEN, D.E.; CARSON, W.G.; TRUMBLE, J.T.; MUTSCHLER, M.A. Acylsugars of wild tomato Lycopersicon pennellii alters settling and reduces oviposition of Bemisia argentifolii (Homoptera: Aleyrodidae). Journal of Economic Entomology, v.88, p.742-748, 1995.

MALUF, W.R.; CAMPOS, G.A.; CARDOSO, M. das G. Relationships between trichome types and spider mite (Tetranychus evansi) repellence in tomatoes with respect to foliar zingiberene contents. Euphytica, v.121, p.73-80, 2001.

PERALTA, I.E.; KNAPP, S.; SPOONER, D.M. New species of wild tomatoes (Solanum Section Lycopersicon: Solanaceae) from northern Peru. Systematic Botany, v.30, p.424-434, 2005.

PEREIRA, G.V.N.; MALUF, W.R.; GONÇALVES, L.D.; NASCIMENTO, I.R. do; GOMES, L.A.A.; LICURSI, V. Seleção para alto teor de acilaçúcares em genótipos de tomateiro e sua relação com a resistência ao ácaro-vermelho (Tetranychus evansi) e à traça (Tuta absoluta). Ciência e Agrotecnologia, v.32, p.996-1004, 2008.

RESENDE, J.T.V. de; MALUF, W.R.; CARDOSO, M.D.; FARIA, M.V.; GONÇALVES, L.D.; NASCIMENTO, I.R. do. Resistance of tomato genotypes with high level of acylsugars to Tetranychus evansi Baker \& Pritchard. Scientia Agricola, v.65, p.31-35, 2008.

SILVA, V.F. Resistência a artrópodos-praga em genótipos de tomateiro ricos em zingibereno e/ou acilaçúcares. 2009. 62p. Tese (Doutorado) - Universidade Federal de Lavras, Lavras.

SUINAGA, F.A.; CASALI, V.W.D.; SILVA, D.J.H. da; PICANÇO, M.C. Dissimilaridade genética de fontes de resistência de Lycopersicon spp. a Tuta absoluta (Meyrick, 1917) (Lepidoptera: Gelechiidae). Revista Brasileira de Agrociência, v.9, p.371-376, 2003.

VILLAS BÔAS, G.L.; FRANÇA, F.H.; MACEDO, N. Potencial biótico da mosca-branca Bemisia argentifolii a diferentes plantas hospedeiras. Horticultura Brasileira, v.20, p.71-79, 2002.

WESTON, P.A.; SNYDER, J.C. Thumbtack bioassay: a quick method of measuring plant resistance to two-spotted spider mites (Acari: Tetranychidae). Journal of Economic Entomology, v.83, p.500-504, 1990.

Recebido em 20 de fevereiro de 2009 e aprovado em 16 de setembro de 2009 